\title{
Orthotopic ileal and sigmoid neobladder after radical cystectomy: functional results: A prospective study and retrospective study
}

\author{
Saied A.A. Mostafa ${ }^{1}$, Ismail M. Khalaf ${ }^{1}$, Ali M.H. Farag ${ }^{1}$, Hassan A. Abdel-Baky ${ }^{1}$, Mostafa \\ M.M. Shakweer ${ }^{2}$, Tarek M. Omran ${ }^{3}$ \\ Departments of ${ }^{1}$ Urology, Radiology ${ }^{2}$ and Clinical Pathology ${ }^{3}$, Faculty of Medicine, Al-Azhar \\ University, Egypt
}

\begin{abstract}
Background: bladder cancer is common among genitourinary tract. Radical cystectomy with urinary diversion is the widely accepted treatment. However, no consensus exists regarding the best orthotopic neobladder creation intestinal segment.
\end{abstract}

Aim of the work: to evaluate functional and oncological outcome of ileal and sigmoid orthotopic urinary diversion.

Patients and methods: The study included two groups of 40 patients in each group (ileal and sigmoid groups). Each group was further divided in two twenty subgroups; prospective and retrospective. Ileal group operated in Urology and Nephrology centre (UNC) and Al-Azhar University hospital (Damietta), while patients in sigmoid group were operated in Al-Azhar University hospital (Cairo). All underwent full history taking, clinical examination, and laboratory investigations. Then, postoperatively, all were followed up for 6 months. In each postoperative follow up visit, patients were assessed clinical, radiological, urodynamic study and by laboratory investigations and patients' quality of life were evaluated.

Results: In ileal orthotopic diversion, early postoperative complications were bleeding (2.5\%), urine leakage $(2.5 \%)$ and wound infection (25\%). Diurnal continence was achieved in $90 \%$, while nocturnal continence was achieved in $70 \%$. The early postoperative complications were urine leakage $(2.5 \%)$, obstruction (2.5\%), and ileus (12.5\%). The late complications were DVT (2.5\%) and intestinal obstruction (5.0\%). In addition, $2.5 \%$ were died from disease. Tumor recurrence was pelvic in $7.5 \%$, distant (hepatic) in $2.5 \%$. Only $25 \%$ of patients were potent and urine culture revealed E-coli in $40.0 \%$. In the sigmoid orthotopic diversion group, the early postoperative complications were urine leakage (17.5\%), obstruction (2.5\%), ileus (7.5\%), bleeding (2.5\%), DVT (2.5\%) and faecal leak (2.5\%). Late complications were pouch stones $(10.0 \%)$, stricture urethra $(12.5 \%)$, ureterocolic stricture $(7.5 \%)$, pouch stones plus stricture urethra in 5.0\%, DVT (2.5\%), intestinal obstruction (10.0\%) and hematemesis in $2.5 \%$. In addition, $12.5 \%$ were died. Tumor recurrence was pelvic in $25.0 \%$. Only $7.5 \%$ of patients were potent and urine culture revealed E-coli in $35.0 \%$. Diurnal continence was reported in $92.5 \%$, while nocturnal continence was reported in $60 \%$.

Conclusion: both sigmoid and ileal orthotopic neobladder provided a satisfactory outcome. Sigmoid neobladder was associated with lower rate of complications and better functional outcome. However, there were many late-term complications detected in this group.

Keywords: bladder cancer, urinary diversion, orthotopic neobladder, sigmoid, ileum.

\section{Introduction}

Bladder cancer is a common type of genitourinary cancer. Radical cystectomy with urinary diversion is the most effective local surgical intervention for invasive bladder cancer. However, radical cystectomy is associated with frequent complications (19$64 \%)^{(1)}$.

After radical cystectomy (RC), the orthotopic neobladder (ON) is the chosentechnique of urinary diversion $^{(2)}$.

$$
\text { Various intestinal segments }
$$
andtechniques have been explored andused for
ON reconstruction. Ileumand sigmoid, the most common substitutesfor an $\mathrm{ON}$, and ileal neobladder (IN) and sigmoid neobladder (SN) were seen to be suitable alternativesto $\mathrm{ON}$ with IN providing better continence and sigmoid neobladder confer better voiding. However, these neobladders are often created by the detubularization technique, occasionally with complex reconfiguration, and they are timeconsuming ${ }^{(3)}$. However, urinary diversion had many complications (e.g., metabolic acidosis, electrolyte imbalance, bacterial growth, 
infection, urolithiasis and renal dysfunction $)^{(4)}$.

As regards quality of life (QoL), including urinary, sexual and social function, daily activities and body image satisfaction, orthotopic continent, diversions are considered the 'gold standard' among reconstructive procedures ${ }^{(5)}$. However, there was no consensus on

\section{Patients and methods}

This study included two patient's setups, one is prospective and the other is retrospective evaluation. The study compromises two group of patients 40 patients in each group. Group I: was again divided in two twenty prospective and another retrospective operated in Urology and Nephrology centre (UNC) and Al-Azhar University hospital (Damietta). Group II: Included also two sets of patients each of them had twenty patients in both equally divided twenty patients in each. The patients in this group were operated in Al-Azhar University hospital (Cairo). The Study period was from January 2015 to January 2016.

Inclusion criteria included the following: Informed consent for participation in the study (for the prospective groups); patients were less than 70 years; no female patients were included; good general condition and health status including renal, cardiac and hepatic profile.

Group I patients underwent ileal neobladder following Hautmann technique as described by Hautmann ${ }^{(6)}$. After surgical intervention, the transurethral catheter needs to be washed with $60 \mathrm{~mL}$ of saline every $6 \mathrm{~h}$. The ureteric catheters were removed 13 and 14 days after surgery, following confirmation that the ileo-ureteric anastomoses were watertight. A quinolone antibiotic is used as prophylaxis for 2 weeks. The patient is taught to void while seated and to slowly extend the neobladder volume to $500 \mathrm{~mL}$, which usually assures continence.

Group II: perceived sigmoid orthotopic neobladder as described by Reddy et $\boldsymbol{a l}^{\left({ }^{(7)} \text {. For }\right.}$ those who were assigned for sigmoid neobladder, adequate anatomical configuration of sigmoid and descending colon obtained via water soluble contrast enema and or colonoscopy if needed.

In this study, the main concern is follow up and evaluation of patients after being discharged from the hospital. The follow up program was scheduled to see the patient in the ideal procedure.

\section{Aim of the work}

It is to evaluate functional and oncological outcome of ileal and sigmoid orthotopic urinary diversion.

outpatient service at 3,6 months and at one year. In each visit, the patient underwent detailed physical examination including the history of voiding pattern, issue of continence, clinical evidence of metabolic acidosis and bowel habits. Laboratory investigations were carried out in each visit including blood picture, serum electrolytes, acid-base profile, renal and hepatic function tests. Radiological evaluation including abdomino-pelvic ultrasonography screening in each visit with careful attention of the hydronephrosis and the presence of significant post-voiding residual urine, computed tomography (CT) scan with contrast for the evidence of oncological failure or tumor recurrence and evaluation of the integrity of the upper tract in addition to screening of the other intra-abdominal abnormalities. Renography was required in cases of suspected obstructed renal unit.

In concern of suspicious of skeletal bony complaints not responding to simple nonsteroidal anti-inflammatory drugs, bone scan was required in such conditions. It is worthily to identify the definition of continence in patients with orthotopic neobladder as complete dryness during daytime and using maximum one protective bad during night time without use of any medications. The study was approved by the Ethics Board of Al-Azhar University.

Statistical analysis of data: The collected data analysed by statistical package for social science (SPSS) version 16 (SPSS Inc., USA). Arithmetic mean and standard deviation were calculated for quantitative data while frequency and distribution were calculated for qualitative data.

\section{Results}

\section{I- Results of ileal orthotopic diversion}

In ileal orthotopic diversion groups, patient age ranged from 38 to 69 years; the mean age was $62.22 \pm 7.45$. Associated medical diseases was reported in $20 \%$ of all studied populations: chronic liver disease in $2.5 \%$, heart disease in $2.5 \%$, diabetes mellitus in $10 \%$ and 
hypertension in $5.0 \%$. The most common pathological tumor stage was T3b (70.0\%) and the most common histological type of tumor was TCC $(72.5 \%)$. The majority of tumors were of high grade $(77.5 \%)$ and lymph node involvement was reported in minority of patients $(10.0 \%)$.

As regard to perioperative data in ileal orthotopic diversion, blood transfusion was reported in $72.5 \%$ of all studied patients, and units of transfused blood ranged from 1 to 2 , with a mean of $1.13 \pm 0.35$. The duration of hospital stays ranged from 8 to 36 days with a mean of $16.72 \pm 6.02$ days. Early postoperative complications were in the form of bleeding in $2.5 \%$, urine leakage in $2.5 \%$ and wound infection in $25 \%$. The clavianDindo grade was 0 in $15 \%, 1$ in $7.5 \%, 2$ in $5 \%$ and $3 \mathrm{a}$ in $2.5 \%$ (table 1).

In the ileal orthotopic diversion group, diurnal continence was achieved in $90 \%$ of patients at 6 months, while nocturnal continence was achieved in $70 \%$ of studied patients.

Results of EORTC QLQ-C30 revealed that, the global score ranged from 3 to 7 ; the mean value was $5.08 \pm 1.16$. The mean physical function score was $1.59 \pm 0.53$ (range 1-3), the mean role function score was $1.79 \pm 0.69$ (range $1-4)$; the mean emotional function was $1.09 \pm 0.27$ (range 1-2), cognitive function score was $1.13 \pm 0.27$ (range 1-2), the mean social function was $1.61 \pm 0.57$ (range 1-3). These data indicated that of function scales, the physical function, cognitive and social functions were the mainly affected scales. In addition, the most common high symptoms score was appetite loss, nausea and vomiting and pain.

As regard to complications among ileal orthotopic diversion group, the early postoperative urologic complications were in the form of urine leakage in $2.5 \%$ and obstruction in $2.5 \%$. The early non-urologic complications were in the form of ileus in $12.5 \%$ and no bleeding, DVT or faecal leak. Uretero-ileal obstruction was bilateral in $2.5 \%$. The late urologic complications were in the form of stricture urethra in 5.0\%, uretero-ileal stricture in $5 \%$ and pouch stones plus stricture urethra in $2.5 \%$. The Orthotopic complications were in the form of stricture urethra in $5.0 \%$. Finally, the late non-urologic complications were in the form of DVT in $2.5 \%$ and intestinal obstruction in $5.0 \%$ (table 2).

As regard to follow up data among ileal orthotopic diversion group, $2.5 \%$ were died from disease, while $90.0 \%$ were living and free and $7.5 \%$ living with disease. Tumor recurrence was pelvic in $7.5 \%$, distant (hepatic) in $2.5 \%$. Postoperative creatinine was above 2 in $2.5 \%$ and postoperative clinical acidosis was reported in $7.5 \%$. Only $25 \%$ of patients were potent and urine culture revealed E-coli in $40.0 \%$, Klebsiella in $2.5 \%$ and proteus in $5.0 \%$. finally, diarrhoea was reported in $10.0 \%$.

As regard to postoperative urodynamic study among ileal orthotopic diversion group, the $\mathrm{Q}_{\max }$ ranged from 7.90 to 29.10, with a mean value of $16.91 \pm 4.99$. Pouch pressure waves were frequent (high) in $2.5 \%$, frequent (low) in $30.0 \%$ and occasional in $30.0 \%$. Residual urine was $<100$ in $60 \%$, and $>100$ in $2.5 \%$. Neobladder capacity was around $450 \mathrm{cc}$ (table 3 ).

Table (1): Perioperative data in ileal orthotopic diversion

\begin{tabular}{|l|l|c|}
\hline \multicolumn{2}{|c|}{ Variable } & Statistics \\
\hline \multirow{3}{*}{ Blood transfusion } & No & $11(27.5 \%)$ \\
\cline { 2 - 3 } & Yes & $29(72.5 \%)$ \\
\cline { 2 - 3 } & Number of transfused units & $1.13 \pm 0.35 ; 1-2$ \\
\hline Duration of hospital stay & None & $16.72 \pm 6.02 ; 9-36$ \\
\hline \multirow{3}{*}{$\begin{array}{l}\text { Early PO } \\
\text { complications }\end{array}$} & bleeding & $28(70.0 \%)$ \\
\cline { 2 - 3 } & Urine leakage & $1(2.5 \%)$ \\
\cline { 2 - 3 } & wound infection & $1(2.5 \%)$ \\
\hline \multirow{3}{*}{ ClavianDindo grade } & None & $10(25.0 \%)$ \\
\cline { 2 - 3 } & Grade 0 & $28(70.0 \%)$ \\
\cline { 2 - 3 } & Grade 1 & $6(15.0 \%)$ \\
\cline { 2 - 3 } & Grade 2 & $3(7.5 \%)$ \\
\cline { 2 - 3 } & Grade 3a & $2(5.0 \%)$ \\
\hline
\end{tabular}

Table (2): Postoperative complications among ileal orthoptic diversion group Variable 


\begin{tabular}{|c|c|c|c|}
\hline \multirow{2}{*}{$\begin{array}{l}\text { Early postoperative } \\
\text { Urologic complications }\end{array}$} & Leakage & 1 & $2.5 \%$ \\
\hline & Obstruction & 1 & $2.5 \%$ \\
\hline \multirow{4}{*}{$\begin{array}{l}\text { Early postoperative } \\
\text { Non-urologic } \\
\text { complications }\end{array}$} & Ileus & 5 & $12.5 \%$ \\
\hline & Bleeding & 0 & $0.0 \%$ \\
\hline & DVT & 0 & $0.0 \%$ \\
\hline & Faecal leak & 0 & $0.0 \%$ \\
\hline \multirow[t]{3}{*}{ Ureterocolic obstruction } & Right uretero-ileal obstruction & 0 & $0.0 \%$ \\
\hline & Left uretero-ileal obstruction & 0 & $0.0 \%$ \\
\hline & Bilateral obstruction & 1 & $2.5 \%$ \\
\hline \multirow{4}{*}{$\begin{array}{l}\text { Late urologic } \\
\text { Complications }\end{array}$} & Pouch stones & 0 & $0.0 \%$ \\
\hline & Stricture urethra & 2 & $5.0 \%$ \\
\hline & Uretero-ileal stricture & 2 & $5.0 \%$ \\
\hline & Pouch stones plus stricture urethra & 1 & $2.5 \%$ \\
\hline \multirow{3}{*}{$\begin{array}{l}\text { Orthotopic } \\
\text { complications }\end{array}$} & Stone (small) & 0 & $0.0 \%$ \\
\hline & Stone (large) & 0 & $0.0 \%$ \\
\hline & Stricture urethra & 2 & $5.0 \%$ \\
\hline \multirow{3}{*}{$\begin{array}{l}\text { Late non-urologic } \\
\text { complications }\end{array}$} & DVT & 1 & $2.5 \%$ \\
\hline & Intestinal obstruction & 2 & $5.0 \%$ \\
\hline & Hematemesis & 0 & $0.0 \%$ \\
\hline
\end{tabular}

Table (3): PO urodynamic study among orthotopic sigmoid diversion group

\begin{tabular}{|l|l|c|c|}
\hline \multicolumn{2}{|c|}{} & $\mathrm{n}$ & $\%$ \\
\hline Q max & \multicolumn{1}{|c|}{$16.91 \pm 4.99 ; 7.90-29.10$} \\
\hline \multirow{4}{*}{ Pouch pressure waves } & Data not available & 15 & $37.5 \%$ \\
\cline { 2 - 4 } & Frequent (high) & 1 & $2.5 \%$ \\
\cline { 2 - 4 } & Frequent (low) & 12 & $30.0 \%$ \\
\cline { 2 - 4 } & Occasional & 12 & $30.0 \%$ \\
\hline \multirow{3}{*}{ Residual urine } & Data not available & 15 & $37.5 \%$ \\
\cline { 2 - 4 } & $<100$ & 24 & $60.0 \%$ \\
\cline { 2 - 4 } & $>100$ & 1 & $2.5 \%$ \\
\hline
\end{tabular}

\section{II- Results of sigmoid orthotopic diversion}

In the sigmoid orthotopic diversion group, patient age ranged from 28 to 69 , the mean age was $52.30 \pm 9.40$ years. The preoperative condition of the kidney was normal in $82.5 \%$ and dilated in $17.5 \%$. teniectomy was done in $77.5 \%$ and not done in $22.5 \%$.

In sigmoid orthotopic diversion, the right ureter anastomosis was submucous tunnel in $75 \%$, teniectomy trough in $17.5 \%$ and direct nipple in 5\%; the right ureter pathology was absent in 55\%, it was thick in $17.5 \%$ and dilated in $17.5 \%$. On the other hand, left ureterocolic anastomosis was submucous in $45 \%$, teniectomy trough in $37.5 \%$ and direct nipple in $17.5 \%$. Furthermore, left ureter pathology was absent in $45 \%$, thick in $25 \%$, and dilated in $22.5 \%$ (table 4 ).

In sigmoid orthotopic diversion group, the most common tumor stage was PT3a (42.5\%) followed by PT3b in $37.5 \%$. The most common tumor type was TCC in $52.5 \%$; the tumor grade was low in 50\% and high in 50\%; and lymph node involvement was reported in $10.0 \%$.
As regard to complications among sigmoid orthotopic diversion group, the early postoperative urologic complications were in the form of urine leakage in $17.5 \%$ and obstruction in $2.5 \%$. The early non-urologic complications were in the form of ileus in $7.5 \%$, bleeding in $2.5 \%$, DVT in $2.5 \%$ and faecal leak in $2.5 \%$. Ureterocolic obstruction was on the right ureter in $10 \%$, left ureter in $7.5 \%$ and bilateral in $2.5 \%$. The late urologic complications were in the form of pouch stones in $10.0 \%$, stricture urethra in $12.5 \%$, ureterocolic stricture in $7.5 \%$ and pouch stones plus stricture urethra in $5.0 \%$. The Orthotopic complications were in the form of small stone in $2.5 \%$, large stone in $2.5 \%$ and stricture urethra in $2.5 \%$. Finally, the late non-urologic complications were in the form of DVT in $2.5 \%$, intestinal obstruction in $10.0 \%$ and hematemesis in $2.5 \%$ (table 5).

As regard to follow up data among sigmoid orthotopic diversion group, $12.5 \%$ were died, $10 \%$ from disease and $2.5 \%$ from unknown causes, while $67.5 \%$ were living and free and $20 \%$ living with disease. Tumor 
recurrence was pelvic in $25.0 \%$, distant (hepatic) in 5\% and pelvic plus distant in 5.0\%. Postoperative creatinine was above 2 in $7.5 \%$ and postoperative clinical acidosis was reported in $5 \%$. Only $7.5 \%$ of patients were potent and urine culture revealed E-coli in $35.0 \%$, Klebsiella in $2.5 \%$ and proteus in $7.5 \%$. finally, constipation was reported in $12.5 \%$.

In sigmoid orthotopic diversion group, the diurnal continence was reported in $92.5 \%$, while nocturnal continence was reported in $60 \%$. The diurnal frequency ranged from 4 to 10 with a mean of $6.25 \pm 1.72$; while nocturnal frequency ranged from 1 to 10 , the mean values were $3.55 \pm 1.78$ (Table 6).

As regard to renal unit condition postoperatively, it was normal in $75 \%$, showed mild dilatation in $2.5 \%$ and significant dilatation in $22.5 \%$.

The postoperative ascending cystogram among orthotopic sigmoid diversion group revealed that, no reflux was reported in $81.25 \%$ of renal units, while reflux was reported in $18.75 \%$ of renal units; the reflux was grade 1 in $7.5 \%$, grade 2 in $3.75 \%$ and grade 3 in $7.5 \%$ of renal units.

As regard to postoperative urodynamic study among orthotopic sigmoid diversion group, the Qmax ranged from 5.80 to 28.60, with a mean value of $14.41 \pm 5.58$. Pouch pressure waves were absent in $15 \%$, frequent (high) in $7.5 \%$, frequent (low) in $30.0 \%$ and occasional in $2.5 \%$. Finally, residual urine was none in $40 \%,<100$ in $20 \%$, and $>100$ in $10 \%$. Neo-bladder capacity was around $520 \mathrm{cc}$.

Table (4): Implantation of ureter among sigmoid orthotopic diversion group

\begin{tabular}{|l|l|c|c|}
\hline \multicolumn{2}{|c|}{ Variable } & $\mathrm{n}$ & $\%$ \\
\hline \multirow{3}{*}{$\begin{array}{l}\text { Right ureter } \\
\text { Anastomosis }\end{array}$} & Inapplicable & 1 & $2.5 \%$ \\
\cline { 2 - 4 } & Submucous tunnel & 30 & $75.0 \%$ \\
\cline { 2 - 4 } & Teniectomy trough & 7 & $17.5 \%$ \\
\cline { 2 - 4 } & Direct nipple & 2 & $5.0 \%$ \\
\hline \multirow{2}{*}{$\begin{array}{l}\text { Right ureter } \\
\text { Pathology }\end{array}$} & None & 22 & $55.0 \%$ \\
\cline { 2 - 4 } & Thick & 7 & $17.50 \%$ \\
\cline { 2 - 4 } & Dilated & 7 & $17.50 \%$ \\
\hline \multirow{2}{*}{$\begin{array}{l}\text { Left ureterocolic } \\
\text { Anastomosis }\end{array}$} & Submucous & 18 & $45.0 \%$ \\
\cline { 2 - 4 } & Teniectomy trough & 15 & $37.5 \%$ \\
\cline { 2 - 4 } & Direct-nipple & 7 & $17.5 \%$ \\
\hline \multirow{2}{*}{$\begin{array}{l}\text { Left ureter } \\
\text { pathology }\end{array}$} & None & 18 & $25.0 \%$ \\
\cline { 2 - 4 } & Thick & 10 & $25.0 \%$ \\
\cline { 2 - 4 } & Dilated & 9 & $22.5 \%$ \\
\hline
\end{tabular}

Table (5): Postoperative complications among sigmoid orthotopic diversion group

\begin{tabular}{|c|c|c|c|}
\hline & Variable & $\mathrm{n}$ & $\%$ \\
\hline Early postoperative & Leakage & 7 & 17.5 \\
\hline Urologic complications & Obstruction & 1 & 2.5 \\
\hline Early postoperative & Ileus & 3 & 7.5 \\
\hline Non-urologic & Bleeding & 1 & 2.5 \\
\hline complications & DVT & 1 & 2.5 \\
\hline & Faecal leak & 1 & 2.5 \\
\hline Ureterocolic obstruction & Right ureterocolic obstruction & 4 & 10.0 \\
\hline & Left ureterocolic obstruction & 3 & 7.5 \\
\hline & Bilateral obstruction & 1 & 2.5 \\
\hline Late urologic & Pouch stones & 4 & 10.0 \\
\hline Complications & Stricture urethra & 5 & 12.5 \\
\hline & Ureterocolic stricture & 3 & 7.5 \\
\hline & Pouch stones plus stricture urethra & 2 & 5.0 \\
\hline Orthotopic & Stone (small) & 1 & 2.5 \\
\hline complications & Stone (large) & 1 & 2.5 \\
\hline & Stricture urethra & 1 & 2.5 \\
\hline Late non-urologic & DVT & 1 & 2.5 \\
\hline complications & Intestinal obstruction & 4 & 10.0 \\
\hline & Hematemesis & 1 & 2.5 \\
\hline
\end{tabular}


Table (6): Continence and frequency among orthotopic sigmoid diversion group

\begin{tabular}{|l|l|c|c|}
\hline \multirow{2}{*}{ Diurnal } & Continent & $\mathrm{n}$ & $\%$ \\
\cline { 2 - 4 } & Stress incontinence & 37 & $92.5 \%$ \\
\hline \multirow{3}{*}{ Nocturnal } & Continent & 3 & $7.5 \%$ \\
\cline { 2 - 4 } & Incontinent & 24 & $60.0 \%$ \\
\cline { 2 - 4 } & hypercontinence & 14 & $35.0 \%$ \\
\hline \multicolumn{2}{|l|}{ Diurnal frequency } & \multicolumn{2}{|c|}{$6.25 \pm 1.72 ; 4-10$} \\
\hline \multicolumn{2}{|l|}{ Nocturnal frequency } & \multicolumn{2}{|c|}{$3.55 \pm 1.78 ; 1-10$} \\
\hline
\end{tabular}

\section{Discussion}

The functional resultsafter the orthotopic neobladder reforming, such as voiding pattern and continence status, have been extensively studied ${ }^{(8)}$. However, there is still controversy regarding which type of neobladder offers the most favourable outcome and patient's satisfaction. Therefore, the present study was designed to evaluate functional and oncological outcome of ileal and sigmoid orthotopic urinary diversion.

In ileal orthotopic diversion, blood transfusion was reported in $72.5 \%$ of all studied patients, and units of transfused blood ranged from 1 to 2 , with a mean of $1.13 \pm 0.35$. The duration of hospital stays ranged from 8 to 36 days with a mean of $16.72 \pm 6.02$ days. Early postoperative complications were in the form of bleeding in $2.5 \%$, urine leakage in $2.5 \%$ and wound infection in $25 \%$. The clavianDindo grade was 0 in 15\%, 1 in $7.5 \%, 2$ in $5 \%$ and $3 \mathrm{a}$ in $2.5 \%$. These results are comparable to Yadav et $\boldsymbol{a l} .{ }^{(9)}$ who reported that, early complications were seen in 12 patients out of 42 patients (28.6\%), whereas late complications were observed in 5 patients (12\%). Among early complications, grades 1 (2.4\%), $2(4.8 \%)$, and $3(7.1 \%)$ complications were observed in 8 $(19 \%), 3(7.1 \%)$, and $1(2.4 \%)$ patient, respectively. No grade 4 or 5 complications were reported. The most common complications related to the surgical wound. Fever needs the change of antibiotics reported in 2 patients $(4.8 \%)$ without any proof of pyelonephritis. One patient required diverting ileostomy for ileal anastomotic leakage.

In the present work, in the ileal orthotopic diversion group, diurnal continence was achieved in $90 \%$ of patients at 6 months, while nocturnal continence was achieved in $70 \%$ of studied patients. Continence after ileal orthotopic diversion depends on multiple factors, the most important is an intact striated sphincter and formation of a low-pressure compliant urinary reservoir ${ }^{(10)}$. Daytime continence rates are around $80 \%-100 \%$, but nighttime continence have a tendency to be much lower, with reports ranging from $45 \%$ to $90 \%{ }^{(11)}$. However, this disparity may be due to inconsistencies (difference between lower and higher reported percentages) and variations in definition of continence ${ }^{(12)}$.

Also, the duration at the time of assessment is vital as continence rates do improve with time ${ }^{(13)}$. Aleksic et al. ${ }^{(14)}$ reported satisfactory continence rates of $71 \%$ at 3 months and $81.6 \%$ at 12 months. In addition, Yadav $\boldsymbol{e t}$ $\boldsymbol{a l}{ }^{(9)}$ found that in postoperative period, most patients had some grade of incontinence, which progressively improved with time as the pouch matured.

Nighttime continence has been relatively difficult to achieve, probably due to loss of local spinal reflex arc, decreased rhabdosphincter tone, and nocturnal diuresis ${ }^{(15)}$. El Bahnasawy et al. ${ }^{(\mathbf{1 2})}$ found that PVR, frequency and maximum amplitude of uninhibited contractions significantly affected nocturnal continence.

In the present work, postoperative wound infection was reported in $25.0 \%$ of ileal group, and this complication was confined to this group. The possible causes of increased such complication included the following: the patient is usually older in age. Moreover, this surgery often involves major blood loss, so autologous blood often needs to be stored preoperatively for transfusion to avoid homologous blood transfusion, especially for immune-compromised patients at specific risk of the existence of post-operative infections, because severe anemia could lead to severe infectious adverse events post-operatively ${ }^{(16)}$. In addition, surgical time for radical cystectomy with orthotopic neobladder urinary diversion by means of the bowel is longer than other urological surgeries ${ }^{(17)}$ and the cases often 
involve older patients with various risk factors or complications with the potential to cause post-operative infections ${ }^{(16)}$.

In ileal group, results of quality of life scales reported acceptable levels on different functional pivots. In addition, the most common high symptoms score was appetite loss, nausea and vomiting and pain. These results agree with Gakis and Stenzl ${ }^{(18)}$ who reported that, the orthotopic neobladder substitution (OBS) is the chosen diversion after $\mathrm{RC}$ and better than all other forms of diversion in terms of better QoL. In addition, Arata et $\boldsymbol{a l} .{ }^{(19)}$ reported that European Organization for Research and Treatment (EORTC) QLQ-C30 functional evaluation showed no significant differences in any items between ileal and sigmoid subgroups; and in contradiction to results of the present work, they reported that diarrhoea was recognized as being worse in the ileal group. However, our results agree with Kramer et al. (20) who reported that $46 \%$ of patient's experience changes in bowel habit after radical cystectomy but only $26 \%$ of patients reported experiencing related dissatisfaction. Primary cause of dissatisfaction was diarrhea and stool loss.

In addition, an interesting study done by Honeck $\boldsymbol{e t}$ al. ${ }^{(21)}$ reported that, regarding defecation frequency, significant differences could be observed between the two groups. Patients with a sigmoid neobladder had a mean defecation frequency of $1.1 /$ day, whereas patients with ileal neobladder had a frequency of 3.1/day. They added, the reduced defecation frequency is one of the main advantages of a sigmoid neobladder

In sigmoid group, the mortality rate in the present work was $12.5 \%$ (5 patients) (10\% died from the disease and $2.5 \%$ died from other unknown aetiology). This percentage is higher than those reported in previous work by Quek et $\boldsymbol{a l} .{ }^{(22)}$ who reported overall mortality rate to be $2.0 \%$. The high mortality rate in the present work can be attributed to older age and advanced disease and high recurrence rate in such patients. Also, Miyake et al. ${ }^{(\mathbf{2 3})}$ reported that, two men $(2.4 \%)$ died perioperatively from pulmonary emboli or liver dysfunction in their patients who underwent sigmoid orthotopic neobladder after radical cystectomy for bladder cancer. Furthermore, different studies reported rates were $1-3 \%^{(24)}$.
As regard to complications among sigmoid orthotopic diversion group, the early postoperative urologic complications were in the form of urine leakage in $17.5 \%$ and obstruction in $2.5 \%$. The early non-urologic complications were in the form of ileus in $7.5 \%$, bleeding in $2.5 \%$, DVT in $2.5 \%$ and faecal leak in $2.5 \%$. Ureterocolic obstruction was on the right ureter in $10 \%$, left ureter in $7.5 \%$ and bilateral in $2.5 \%$. The late urologic complications were in the form of pouch stones in $10.0 \%$, stricture urethra in $12.5 \%$, ureterocolic stricture in $7.5 \%$ and pouch stones plus stricture urethra in $5.0 \%$. the Orthotopic complication were in the form of small stone in $2.5 \%$, large stone in $2.5 \%$ and stricture urethra in $2.5 \%$. Finally, the late non-urologic complications were in the form of DVT in $2.5 \%$, intestinal obstruction in $10.0 \%$ and hematemesis in $2.5 \%$. Miyake et al.$^{(23)}$ reported that,there were 45 early complications in 28 patients $(35 \%)$. The first, second and third most common were wound infection, ileus and pyelonephritis, respectively. Only one need reoperation, due to deep venous thrombus. Late complications were reported in $19 \%$. Neobladder stone, uretero-intestinal stricture and entero-urethral stricture were the most common. Surgical intervention was done for 16 of the 19 late complications.

The results of the present work revealed that, patients in ileal group were significantly older in age, had significant high stage of tumor, but significant lower rate of lymph node involvement. The overall early complications were significantly lower in sigmoid $(17.5 \%)$ versus ileal group (35\%). However, wound infection is restricted to the ileal group. Night continence was higher in sigmoid group. Guan et $\boldsymbol{a l} .{ }^{(25)}$ reported that, the neobladder-related complications were common in both types of orthotopic neobladder renovation. Common early complications included infection, urinary leakage and ileus.

In addition, other reports found that, early complications were higher in sigmoid than in ileal group. In addition, the common late complications included uretero-enteric and vesico-urethral strictures, incisional hernias, neobladder calculi and intestinal obstruction ${ }^{(8)}$.

The possible explanation for reversal of incidence of complications in this study than reported in literature ${ }^{(26)}$ can be attributed that, the sigmoid approach was adopted by Al-Azhar 
University since $1992^{(26)}$; and the work nearly confined to this approach. Thus, the rate of complications decreased by time. However, the ileal approach was introduced more recently and practiced line by line to other approaches to Al-Mansoura Oncology Center ${ }^{(27)}$. The recent instruction of the approach may be responsible to high rate of complications. However, the rate of complications reported after ileal neobladder approach in the present work is less than reported incidence in literature ${ }^{(27)}$, which reflecting the competency and good results in Mansoura Oncology Center.

Furthermore, Tao et al. ${ }^{(28)}$ in their metaanalysis reported that, the overall incidence of late complications was similar between the two groups. Steven and Poulsen ${ }^{(29)}$ reported that, these complications were common in neobladder reconstructions. However, most could be managed conservatively. The incidence could be reduced with the development of procedures and the improvement of surgical techniques.

Among some serious complications related to orthotopic neobladder, voiding dysfunction was an important factor unfavorably affecting the patient's satisfaction. Most previous reportsreinforced that sigmoid neobladder provide a better satisfactory voiding function than ileal neobladder ${ }^{(23)}$.

In the present study, voiding function was assessed after sigmoid orthotopic neobladder reconstruction and revealed that the residual urine was none in $40 \%,<100$ in $20 \%$, and $>100$ in $10 \%$. Neo-bladder capacity was around $520 \mathrm{cc}$, which reflect the good voiding function. It was reported in literature that, sigmoid neobladder could assist more subjects in spontaneous voiding than ileal one. It might be explained by several reasons linked to physiologic properties of the sigmoid. The functions of sigmoid segment could be profusely supplied by nerves of the sacral parasympathetic plexus originating from the S2 to S4 of the spinal $\operatorname{cord}^{(30)}$. For the overextension of thin ileal wall, the weak contraction of new ileal bladder amplified the risk of voiding dysfunction, and the incidence increased with time. In addition, factors due to different voiding state should be evaluated, for example, the experience of surgeons involved in each type of NB creation ${ }^{(31)}$.

Continence status is another significant factor affecting the patient's quality of life.
According to the results of Tao et al. ${ }^{(28)}$ metaanalysis, the incidences of daytime continence in patients with sigmoid and ileal neobladders were $66.7-90 \%$ and $74.1-97 \%$, respectively. The nighttime continence rates ranged from $23.8 \%$ to $65.2 \%$ and from $57.1 \%$ to $90 \%$, respectively. This meta-analysis revealed that patients could achieve better continence status in ileal neobladder group than in sigmoid neobladder group (also this is in contradiction to results of the present work), particularly during the night-time ${ }^{(32)}$.

Most surgeons improved the continence by forming a compliant urinary reservoir from a detubularized intestinal segment and preserving as much of the distal urethral sphincter as possible. The purpose of detubularization is to create a reservoir with high capacity and low pressure. Surgical preparation of sphincter-active membranous urethra has a great influence on continence status, particularly during daytime ${ }^{(28)}$.

Furthermore, Honeck

et $a \mathbf{l}^{(21)}$ conducted a study to compare between ileal and sigmoid orthotopic diversion and concluded that, orthotopic bladder substitution with sigmoid segments has shown equivalent results regarding micturition parameters, continence rates and quality of life compared to orthotopic ileal bladder substitution. One advantage seems to be the reduced defecation frequency in patients with sigmoid neobladder compared to the ileal neobladder. The sigmoid neobladder meets the functional day and night requirements for a bladder substitution and is a useful alternative to the ileal neobladder.

The present work revealed that, on the short-term, sigmoid orthotopic diversion was associated with a better outcome than ileal group. However, on the long-term, results of the sigmoid group showed a high rate of complications. These results agree with Miyake and Fujisawa ${ }^{(33)}$ who reported that, there are some advantages in selecting sigmoid neobladder such as the low incidence of urinary tract infection in its use (as found in the present work). It also has little effect on metabolic and nutritional status and preserves the renal function. In addition, sigmoid neobladder is likely to acquire an adequate voiding status by emptying the reservoir. Furthermore, a small post-void residual urine, which has been shown to influence continence with a neobladder, may also have contributed to the favorable 
continence status in sigmoid neobladder ${ }^{(23,30)}$.

Collectively, these findings suggest that the reconstruction of orthotopic sigmoid neobladder could facilitate at least equivalent or even better clinical outcomes than that of ileal neobladder, particularly in women, considering their favorable postoperative voiding status ${ }^{(33)}$.

\section{Conclusion}

Both sigmoid and ileal orthotopic neobladder provided a satisfactory outcome. On short-term follow up the sigmoid neobladder References

1. Hautmann RE, de Petriconi RC, Pfeiffer C (2012): Radical cystectomy for urothelial carcinoma of the bladder without neoadjuvant or adjuvant therapy: longterm results in 1100 patients. Eur Urol., 61: 1039-1047.

2. Hautmann RE, de Petriconi RC and Volkmer BG (2010): Lessons learned from 1,000 neobladders: the 90-day complication rate. J Urol., 184: 990.

3. Xu K, Liu CX and Zheng SB (2013): Orthotopic Detaenial Sigmoid Neobladder after Radical Cystectomy: Technical Considerations, Complications and Functional Outcomes. J Urol., 190: 928-934.

4. Anderson CB, Cookson MS and Chang SS (2012): Voiding Function in Women with Orthotopic Neobladder Urinary Diversion. J Urol., 188: 200-204.

5. Jeong IG, You D and Kim J (2012): Factors associated with non-orthotopic urinary diversion after radical cystectomy. World $\mathbf{J}$ Urol., 30: 815-820.

6. Hautmann RE (2000): Fifteen years' experience with the ileal neobladder: what have we learned? Urol. A., 40: 360-367.

7. Reddy PK, Lange PH and Fraley EE (1991): Total bladder replacement using detubularized sigmoid colon: technique and results. J Urol., 145: 51-55.

8. Miyake H, Furukawa J, Sakai I et al. (2013): Orthotopic sigmoid vs. ileal neobladders in Japanese patients: a comparative assessment of complications, functional outcomes, and quality of life, Urol. Oncol ., 31: 1155-1160.

9. Yadav SS, Gangkak G, Mathur R et al. (2016): Long-term Functional, Urodynamic, and Metabolic Outcome of a Modified Orthotopic Neobladder Created seems to be associated with lower rate of complications and better functional outcome. However, there were many late-term complications detected in those patients, while in ileal neobladder the long-term outcome was not addressed due to methodological issues. Thus, it is recommended to design future prospective studies to estimate a functional and oncological outcome of both techniques with determination of the same parameters of outcome in both techniques.

with a Short Ileal Segment: Our 5-year Experience. Urology, 94: 167-172.

10. Simon J, Bartsch GJ and Kufer R (2006): Neobladder emptying failure in males: incidence, etiology and therapeutic options. J Urol., 176:1468- 1472.

11. Ahmadi H, Skinner EC, Cai J et al. (2013): Urinary functional outcome following radical cystoprostatectomy and ileal neobladder reconstruction in male patients. J Urol., 189:1782-8.

12. El Bahnasawy MS, Osman Y, Gomha MA et al. (2000): Nocturnal enuresis in men with an orthotopic ileal reservoir: Urodynamic evaluation. J Urol., 164:10-3.

13. Zhang Z, Qi H, Zhou R et al. (2013): Early and late urodynamic assessment of the orthotopic N-shaped neobladder. Oncol Lett., 6:1053-1056.

14. Aleksic $P$, Bancevic $V$, Milovic $N$ et al. (2010): Short ileal segment for orthotopic neobladder: A feasibility study. Int J Urol., 17:768-73.

15. Daneshmand S, Bartsch G (2011): Improving selection of appropriate urinary diversion following radical cystectomy for bladder cancer. Expert Rev Anticancer Ther., 11:941-948.

16. Esposito $S$ (2001): Immune system and surgical site infection. J Chemother., 13:12-6.

17. Takeyama K, Matsukawa M, Hotta $\mathrm{H}$ et al. (2005): Incidence of and risk factors for surgical site infection in patients with radical cystectomy with urinary diversion. J Infect Chemother., 11:177-81.

18. Gakis G, Stenzl A (2010): Ileal neobladder and its variants. Eur Urol Suppl., 9:745-53.

19. Arata R, Saika $T$ and Tsushima $T$ (2007): orthotopic ileal neobladder versus sigmoidal neobladder: a quality of life survey. Acta Med Okayama, 61(4): 229- 
234.

20. Kramer MW, von Klot CA et al. (2015): Long-term bowel disorders following radial cystectomy: an underestimated issue? World J Urol., 33(10):1373-1380

21. Honeck P, Bolenz C, Wendt-Nordahl G et al. (2009): Outcome of Sigmoid Compared to Ileal Orthotopic Bladder Substitution in Terms of Micturition and Defecation Parameters in an 8-Year Long-Term Follow-Up. Urol Int., 83:277-280

22. Quek ML, Stein JP, Daneshmand S et al. (2006): A critical analysis of perioperative mortality from radical cystectomy. J Urol., 175: 886-9.

23. Miyake H, Furukawa J, Takenaka A et al. (2008): Long-term functional outcomes in patients with various types of orthotopic intestinal neobladder. Int. J. Urol., 15: 612615.

24. Studer UE, Burkhard FC and Schumacher M (2006): Twenty years' experience with an ileal orthotopic low pressure bladder substitute-lessons to be learned. J Urol., 176: 161.

25. Guan Z, Li J and Yang R (2013): Impacts of different grafts for bladder reconstruction on postoperative recovery of the patients. Nan Fang Yi $\mathrm{Ke} \mathrm{Da}$ XueXue Bao., 33(8):1250-2.

26. Khalaf I (1992): Long term follow up of continent orthotopic sigmoid pouch urinary diversion, J. Urol., 155, 520-526.

27. Abol-Enein H, Tilki D, Mosbah A et al. (2011): Does the Extent of Lymphadenectomy in Radical Cystectomy for Bladder Cancer Influence Disease-Free Survival? A Prospective Single-Center Study. Euro Urol., 60: 572- 577.

28. Tao S, Long Z, Zhang X et al. (2016): Ileal versus sigmoid neobladder as bladder substitute after radical cystectomy for bladder cancer: A meta-analysis. Int $\mathrm{J}$ Surg., 27: 39-45.

29. Steven K, Poulsen AL (2000): The orthotopic Kock ileal neobladder: functional results, urodynamic features, complications and survival in 166 men. J Urol., 164:288-295.

30. Miyake H, Furukawa J, Muramaki M et al. (2010): Orthotopic sigmoid neobladder after radical cystectomy: assessment of complications, functional outcomes and quality of life in 82 Japanese patients, BJU Int., 106: 412-416.

31. Lee RK, Abol-Enein H, Artibani W et al. (2014): Urinary diversion after radical cystectomy for bladder cancer: options, patient selection, and outcomes, BJU Int., 113: 11-23.

32. Miyake H, Furukawa J, Muramaki M et al. (2012): Health related quality of life after radical cystectomy: comparative study between orthotopic sigmoid versus ileal neobladders, Eur. J. Surg. Oncol., 38: 1089-1094.

33. Miyake H, Fujisawa M (2012): Sigmoid neobladder as an ideal form of orthotopic urinary reconstruction. Int J Urol., 19: 184-186. 